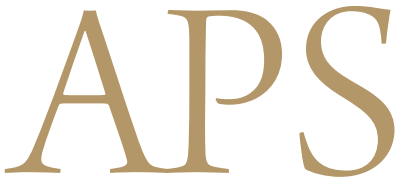

Archives of Plastic Surgery

\title{
Characteristics of adhesion areas between the tissue expander and capsule in implant-based breast reconstruction
}

\author{
Yoon Min Lim, Kwang Hyun Park, Dong Won Lee, Dae Hyun Lew, Tai Suk Roh, Seung Yong Song \\ Department of Plastic and Reconstructive Surgery, Institute for Human Tissue Restoration, Yonsei University College of Medicine, Seoul, Korea
}

Background The use of anatomic implants has improved the aesthetic results of breast surgery; however, implant malrotation is an uncommon, but serious complication of these procedures. Nevertheless, little research has explored implant adhesion. In this study, we investigated adhesion between the expander and the capsule.

Methods Seventy-nine cases of immediate breast reconstruction via two-stage implantbased reconstruction performed between September 2016 and November 2017 were evaluated. Mentor CPX4 expanders were used in 14 breasts, and Natrelle expanders in 65. We analyzed areas of adhesion on the surfaces of the tissue expanders when they were exchanged with permanent implants. We investigated whether adhesions occurred on the cephalic, caudal, anterior, and/or posterior surfaces of the expanders.

Results Total adhesion occurred in 18 cases, non-adhesion in 15 cases, and partial adhesion in 46 cases. Of the non-adhesion cases, 80\% $(n=12)$ were with Mentor CPX4 expanders, while $94.4 \%(n=17)$ of the total adhesion cases were with Natrelle expanders. Of the partial adhesion cases, 90.7\% involved the anterior-cephalic surface. The type of tissue expander showed a statistically significant relationship with the number of attachments in both univariate and multivariate logistic regression analyses $(\mathrm{P}<0.001)$ and with total drainage only in the univariate analysis $(\mathrm{P}=0.015)$.

Conclusions We sought to identify the location(s) of adhesion after tissue expander insertion. The texture of the implant was a significant predictor of the success of adhesion, and partial adhesion was common. The anterior-cephalic surface showed the highest adhesion rate. Nevertheless, partial adhesion suffices to prevent unwanted rotation of the expander.

Keywords Mammaplasty / Breast implants / Breast implantation / Rotation / Tissue expansion devices
Correspondence: Seung Yong Song Department of Plastic and Reconstructive Surgery, Institute for Human Tissue Restoration, Yonsei University College of Medicine, 50-1 Yonsei-ro, Seodaemun-gu, Seoul 03722, Korea

Tel: +82-2-2228-2210

Fax: +82-2-393-6947

E-mail: pssysong@yuhs.ac

Received: 28 Apr 2018 • Revised: 23 May 2019 • Accepted: 29 May 2019

pISSN: 2234-6163 • elSSN: 2234-6171 • https://doi.org/10.5999/aps.2018.00395 • Arch Plast Surg 2019;46:330-335

\section{INTRODUCTION}

The use of anatomical implants has led to improved aesthetic results following certain procedures, including breast recon- struction and breast augmentation [1]. Accurately-shaped anatomic breast implants contain more filling material in the inferior pole of the implant than in the superior pole, in order to imitate the natural shape of the breast as closely as possible. 
An uncommon, but seriously compromising complication that may occur following surgery is rotation of the implant. Because round implants are symmetrical, rotation does not typically cause them to appear malpositioned. However, rotation of anatomic implants can lead to the appearance of a misshapen breast $[2,3]$.

In some studies, the authors have reported a rotation rate ranging from $0.9 \%$ to $14 \%[2,4-9]$. In a 10 -year study by Bengtson et al. [10] and in the study of Cunningham [11], after a 3-year follow-up period, $2.6 \%$ and $1.1 \%$ of primary augmentations, $4.7 \%$ and $2.3 \%$ of augmentation revision procedures, $4.9 \%$ and $2.5 \%$ of primary reconstruction procedures, and $3.0 \%$ and $1.5 \%$ of revision reconstruction procedures demonstrated implant malposition [10].

Numerous factors influence implant rotation [12]. A lack of connective tissue adhesion between the implant and the capsule can occur, prompting movement of the implant in the pocket [5]. Heitmann et al. [13], in their report, found that six of 132 patients experienced implant rotation because the surgical pocket was too large. Other factors that contribute to implant rotation include capsular fluid collection and prosthetic massage $[14,15]$. In addition, double capsules and dynamic action of the muscle, which may induce the implant to move laterally or vertically, also have been reported as possible factors contributing to implant rotation [16,17].

Meanwhile, anaplastic large cell lymphoma (ALCL) has become a major issue in breast augmentation and breast reconstruction surgery. In a recent study, it was reported that ALCL is mainly diagnosed in textured implants and may have some association with the textured surface, although the data remain too preliminary to draw any definitive conclusions [18]. A cumulative review of patients' status showed that ALCL is more prevalent in implants with aggressive texturing [19]. One of the most important reasons for using textured surfaces in anatomically shaped implants is to promote adhesion between the surface of the textured implant and the capsule, which is expected to prevent implant migration [20]. It is well-known that aggressively textured implant surfaces are associated with an increased risk of ALCL, meaning that the relationship between adhesion and ALCL is emerging as a noteworthy research topic.

However, to date, it is not known which parts of the implant are adhesively bonded to tissue, and which factors influence adhesion. In this study, we analyzed the adhesion pattern of tissue expanders, which are not permanent implants, but have similar texturing and are placed in the human body for several months. Thus, we sought to identify the patterns of adhesion development between textured surfaces and tissues in the human body, and which factors promote or degrade it.

\section{METHODS}

From September 2016 to November 2017, 79 tissue expanders were implanted in 75 patients at our facility as part of two-staged implant-based breast reconstruction procedures. Of these 79 tissue expanders, 14 were Mentor CPX4 expanders (Mentor Worldwide LLC, Irvine, CA, USA) and 65 were Natrelle expanders (Allergan plc, Dublin, Ireland). After mastectomy, acellular dermal matrix $(\mathrm{ADM})$ was sutured along the inframammary fold and laterally to the chest wall. The superior part of the $\mathrm{ADM}$ was then sutured to the inferior border of the pectoralis major muscle to create a subpectoralis major muscle and $\mathrm{ADM}$ pocket. In 48 cases, CGCryoDerm (Daewoong Bio Inc., Seoul, Korea) was used, while MegaDerm (L\&C Bio Co., Ltd., Seongnam, Korea) was used in 22 cases and DermACELL (LifeNet Health, Virginia Beach, VA, USA) was used in 10 cases. The size of the $\mathrm{ADM}$ varied from $4 \times 16$ to $6 \times 17 \mathrm{~cm}$. In all cases, the tissue expanders were inserted into the subpectoralis major muscle and acellular ADM pocket. Postoperatively, all patients used a Surgi-Bra (Golda Inc., Cleveland, OH, USA) continuously for 4 weeks and a nonwired sports bra for 2 weeks thereafter.

The presence of an adhesion area between the tissue expander and the capsule was investigated during the second operation, in which the tissue expander was exchanged for a permanent implant. We used nearly the same incision site as the previous mastectomy incision. A designated single operator checked for areas of adhesion between the tissue expander and capsule by dissection with a finger before removing the tissue expander in all cases. We calculated the interval from the immediate tissue expander reconstruction to the time of the second operation. We divided the tissue expanders into four areas: anterior-cephalic, anterior-caudal, posterior-cephalic, and posterior-caudal (Fig. 1), and categorized the patterns of adhesion into three types according to the number of areas that adhered. Total adhesion meant that adhesion had occurred in four areas, partial adhesion meant that adhesion had occurred in one to three areas, and non-adhesion meant that adhesion did not occur in any area. We investigated patient characteristics, such as tissue expander type, age, body mass index, total inflation amount, chemotherapy status, radiotherapy status, total Hemovac drainage amount, Hemovac drain removal date, the brand of $\mathrm{ADM}$, and mastectomy type (total, skin-sparing, or nipple-sparing). Outcomes (number of attachments) were first analyzed with univariate analysis and then with multivariate logistic regression to quantify the impact of relevant patient demographics. Odds ratios were estimated using a logistic regression model. Patient demographics were summarized as means with standard deviations for continuous variables and as median values with interquartile 
ranges $(\mathrm{Q} 1, \mathrm{Q} 3)$ for continuous variables that did not show a normal distribution. Chi-square analysis or the Fisher exact test was used for categorical variables, the Mann-Whitney $U$ test was used for continuous variables that did not show a normal distri-

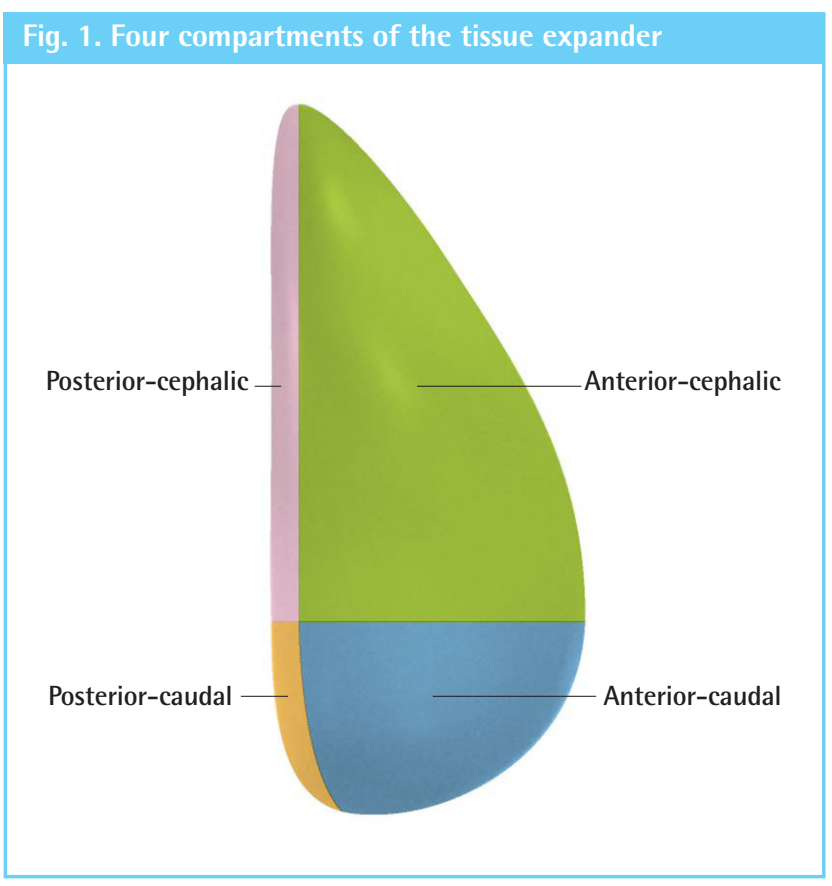

bution, and the independent t-test was used for other continuous variables.

All statistical analyses were conducted using $\mathrm{R}$ version 3.4.4 (The R Foundation for Statistical Computing, Vienna, Austria).

\section{RESULTS}

There were 18 cases of total adhesion, 46 cases of partial adhesion, and 15 cases of non-adhesion. In terms of adhesion location, there were 58 cases of anterior-cephalic adhesion (58/64, $90.6 \%), 43$ cases of anterior-caudal adhesion (43/64, 67.2\%), 41 cases of posterior-cephalic adhesion $(41 / 64,64.1 \%)$, and 26 cases of posterior-caudal adhesion $(26 / 64,40.6 \%)$. There were three cases of minimal infections, which were addressed using oral antibiotics. All three of those infection cases involved a Natrelle expander and showed partial adhesion (posterior-caudal adhesion in one case and anterior-caudal adhesion in two cases). The interval between the first operation and the second operation, in which the tissue expander was exchanged with a permanent breast implant, ranged from 4 months to 22 months (mean, 12 months). In the second operation, in which the expander was converted to a permanent implant, we used an Allergan Natrelle Style 410 implant in 55 cases and a Mentor CPG

\section{Table 1. Demographic and clinical information of the patients}

\begin{tabular}{|c|c|c|c|c|}
\hline \multirow{2}{*}{ Variable } & \multirow{2}{*}{ Total $(n=79)$} & \multicolumn{2}{|c|}{ No. of attachments } & \multirow{2}{*}{ P-value } \\
\hline & & $0(n=15)$ & $\geq 1(n=64)$ & \\
\hline Tissue expander brand & & & & $<0.001$ \\
\hline Allergan & 65 (82.28) & $3(20.00)$ & $62(96.88)$ & \\
\hline Mentor & $14(17.72)$ & $12(80.00)$ & $2(3.12)$ & \\
\hline Age (yr) & $45.54 \pm 9.57$ & $44.07 \pm 8.55$ & $45.89 \pm 9.83$ & 0.510 \\
\hline BMl $\left(\mathrm{kg} / \mathrm{m}^{2}\right)$ & $21.80(20.23-24.34)$ & $20.68(19.73-23.38)$ & $22.18(20.39-24.41)$ & 0.100 \\
\hline Total inflation & $470.00(360.00-520.00)$ & $460.00(370.00-510.00)$ & $470.00(357.50-520.00)$ & 0.970 \\
\hline Chemotherapy & & & & 0.753 \\
\hline No & $34(43.04)$ & $7(46.67)$ & $27(42.19)$ & \\
\hline Yes & $45(56.96)$ & $8(53.33)$ & $37(57.81)$ & \\
\hline Radiotherapy & & & & 0.331 \\
\hline No & 20 (25.32) & $2(13.33)$ & $18(28.12)$ & \\
\hline Yes & 59 (74.68) & $13(86.67)$ & $46(71.88)$ & \\
\hline HV removal date & $19.00(16.50-21.00)$ & $20.00(18.00-21.00)$ & $19.00(15.00-21.00)$ & 0.311 \\
\hline Total HV drainage amount & $2,217.00(2,079.00-2,384.50)$ & $2,365.00(2,246.50-2,437.50)$ & $2,195.00(2,055.25-2,357.75)$ & 0.015 \\
\hline ADM type & & & & $>0.999$ \\
\hline CGcryoDerm & $47(59.49)$ & $9(60.00)$ & $38(59.38)$ & \\
\hline MegaDerm & $22(27.85)$ & $4(26.67)$ & $18(28.12)$ & \\
\hline DermACELL & $10(12.66)$ & $2(13.33)$ & $8(12.5)$ & \\
\hline Interval from first to second operation & $153.00(119.50-284.50)$ & $228.00(167.50-259.00)$ & $137.50(117.00-296.75)$ & 0.111 \\
\hline Mastectomy type & & & & 0.727 \\
\hline Total mastectomy & $24(30.38)$ & $4(26.67)$ & $20(31.25)$ & \\
\hline Skin-sparing mastectomy & $11(13.92)$ & $1(6.67)$ & $10(15.62)$ & \\
\hline Nipple-sparing mastectomy & $44(55.7)$ & $10(66.67)$ & $34(53.12)$ & \\
\hline
\end{tabular}


implant in 24 cases. There was one case of rotation, which was confirmed to show $90^{\circ}$ clockwise rotation at 12 months. This involved a Mentor CPGTM333 430-mL implant (tall height; high projection; width, $12 \mathrm{~cm}$; height, $12.5 \mathrm{~cm}$; projection, $6 \mathrm{~cm}$ ) that showed non-adhesion, which was resolved by capsulectomy and change of the implant to Allergan Natrelle Style 410.

The patients' demographic and clinical information is summarized in Table 1. Seventeen cases of total adhesion were noted with the Natrelle expander, while only one case of total adhesion was noted with the Mentor CPX4 expander. The brand of tissue expander and the total Hemovac drainage amount showed statistically significant relationships with the number of attachments $(\mathrm{P}<0.001$ and $\mathrm{P}=0.015$, respectively). No statistical significance was found for the relationships of the mastectomy type and interval between the first and second operations with the number of attachments. When the cases were stratified by the tissue expander brand, non-adhesion (attachment number $=0$ ) was observed in $20 \%$ of cases using an Allergan Natrelle expander and in $80 \%$ of cases using a Mentor CPX4. The Natrelle expander and Mentor CPX4 showed a significant difference in the number of cases with at least one attachment (96.9\% and $3.1 \%$, respectively) (Table 1 ).

Additionally, in the multivariable logistic results, only the tis- sue expander brand showed a statistically significant relationship with adhesion $(\mathrm{P}<0.001)$. Therefore, the tissue expander brand was found to be the variable with the most influence on the number of attachments (Table 2).

\section{DISCUSSION}

In this study, we divided the areas of the tissue expanders into four categories based on anatomical location, and investigated which areas most frequently adhered to the surrounding tissues. We also studied patient-related factors that affected adhesion and attempted to determine how much adhesion was required to avoid malrotation of the implant.

In implant-based breast reconstruction and breast augmentation, breast implant adhesion refers to fibrosis with the capsule and implant. In a study by Smahel et al. [21], the authors stated that the soft tissue response was different depending on the texture of the implant used. For 6-8 months after implantation, at the microscopic level, the number of myofibroblasts increased as compared with the smooth side and collagen deposition was more progressive in the "bridges" than at the base of villous processes. After 8 months, the basal layer of the capsule was fibrotic. As a result, at the microscopic level, cavities were present in the

\section{Table 2. Logistic regression analysis}

\begin{tabular}{|c|c|c|c|c|}
\hline \multirow{2}{*}{ Variable } & \multicolumn{2}{|c|}{ Univariable logistic regression } & \multicolumn{2}{|c|}{ Multivariable logistic regression } \\
\hline & OR $(95 \% \mathrm{CI})$ & P-value & OR $(95 \% \mathrm{Cl})$ & P-value \\
\hline \multicolumn{5}{|l|}{ Tissue expander brand } \\
\hline Allergan & Reference & & Reference & \\
\hline Mentor & $0.008(0.001-0.054)$ & $<0.001$ & $0.009(0.001-0.063)$ & $<0.001$ \\
\hline Age & $1.021(0.961-1.083)$ & 0.505 & & \\
\hline BMl & $1.242(0.986-1.565)$ & 0.066 & & \\
\hline Total inflation & $0.999(0.994-1.005)$ & 0.845 & & \\
\hline \multicolumn{5}{|l|}{ Chemotherapy } \\
\hline No & Reference & & & \\
\hline Yes & $1.199(0.388-3.709)$ & 0.753 & & \\
\hline \multicolumn{5}{|l|}{ Radiotherapy } \\
\hline No & Reference & & & \\
\hline Yes & $0.393(0.081-1.919)$ & 0.248 & & \\
\hline Hemovac removal date & $0.927(0.831-1.035)$ & 0.179 & & \\
\hline Total Hemovac drainage amount & $0.998(0.996-1.000)$ & 0.036 & $0.999(0.996-1.003)$ & 0.710 \\
\hline \multicolumn{5}{|l|}{ ADM type } \\
\hline CGcryoDerm & Reference & & & \\
\hline MegaDerm & $1.066(0.289-3.928)$ & 0.924 & & \\
\hline DermACELL & $0.947(0.171-5.245)$ & 0.951 & & \\
\hline Interval from first to second operation & $0.999(0.995-1.004)$ & 0.763 & & \\
\hline \multicolumn{5}{|l|}{ Mastectomy type } \\
\hline Total mastectomy & Reference & & & \\
\hline Skin-sparing mastectomy & $2.000(0.197-20.325)$ & 0.558 & & \\
\hline Nipple-sparing mastectomy & $0.680(0.188-2.456)$ & 0.556 & & \\
\hline
\end{tabular}


textured implant surface. They called these cavities "bridges." Fibrosis in these bridges led to better adhesion between the capsule and implant. Based on those results, it could be predicted that more aggressive implant texturing induces more adhesion.

In the current study, we found that the extent to which capsule and tissue expander adhesion was induced depended on the brand of tissue expander used. The Natrelle expander demonstrated more instances of adhesion than did the Mentor CPX4 expander. Due to the differences in the manufacturing process, the Natrelle expander has a more aggressively textured surface than Mentor CPX4, as shown by microscopy [22]. This means that the texture of the implant is associated with adhesion. In a study by Montemurro et al. [12], the authors stated their belief that good adhesive properties contributed to a lower rotation rate. Furthermore, Danino et al. [23] demonstrated that a sufficient texture contributed to a more stable adhesive effect involving the surrounding capsule. A prospective study of electron microscopic findings in adhesion and non-adhesion areas could yield insights into breast implant adhesion [21].

In our study, the anterior part of the expander was the area that was most adhesive to the capsule, and the posterior-caudal part was the area that was most non-adhesive to the capsule. The anterior part of the expander is the area where the least fluid collects, because it is a non-dependent area, while the posteriorcaudal part is the area where the most fluid collects because it is the dependent area of the pocket. Seroma was found to have an effect on adhesion between the implant and capsule [14], suggesting that differences in fluid collection may be a contributing factor to this finding.

There were no instances of malrotation in cases of tissue expander insertion in our study, although there was one case of malrotation that showed non-adhesion after the second operation. Even in the 15 expanders with non-adhesion, malrotation did not occur. A reason for this may be that the observer missed slight malrotation. In addition, it is presumed that the frictional force of the expander itself prevented malrotation, even without adhesion.

Partial adhesion may be a common phenomenon after breast tissue expander insertion, and it is possible that it could occur at a similar rate in cases of breast augmentation or permanent implant placement in breast reconstruction. Although our study had a relatively short duration of observation, and did not observe the actual adhesion of permanent implants with capsules, we predict that even partial adhesion suffices to prevent expander rotation. Because of this limitation, in a further study we will be able to strengthen our argument by incorporating a longer period of observation and visual confirmation of the adhesion of permanent implants with capsules.
In the study of Maxwell et al. [8], appropriate adhesion of the implant to the surrounding tissue was found to be essential for maximizing the benefits of the procedure and securing a low rate of rotation. Maxwell et al. urged surgeons to ensure precise pocket dissection, with the drain inserted into a precisely fitting implant pocket and bloodless sharp dissection (with loose connective tissue left on the ribs to reduce bleeding), and to avoid massage and vigorous displacement exercises for up to 3 months, as those steps may promote tissue adhesion and prevent implant rotation.

However, our study suggests that aggressive texturing is a more important factor than those described by Maxwell et al. The aggressiveness of the texture of the implant was found to be the most important factor associated with implant adhesion in this study. This result is noteworthy, since ALCL also occurs more frequently in aggressively textured implants. As the relationship between ALCL and textured implants is being actively researched, it is expected that further studies of the relationship between ALCL and the extent of adhesion will be of interest and shed light onto basic aspects of ALCL.

In this study, we evaluated the location of adhesion after tissue expander insertion. Our data indicated that the texture of the implant was a significant factor contributing to the success of adhesion. Partial adhesion is a common phenomenon in expander-based breast reconstruction. Nevertheless, partial adhesion was found to be sufficient to prevent unwanted rotation of the implant in this study. Because the anterior-cephalic surface showed the highest adhesion rate, the anterior portion of the implant may have the highest likelihood of successful adhesion to the capsule.

\section{NOTES}

\section{Conflict of interest}

No potential conflict of interest relevant to this article was reported.

\section{Ethical approval}

The study was and performed in accordance with the principles of the Declaration of Helsinki. Written informed consents were obtained.

\section{Author contribution}

Conceptualization: Song SY, Park KH. Data curation: Park KH, Lim YM. Formal analysis: Lim YM. Methodology: Lee DW. Project administration: Lew DH, Roh TS. Writing - original draft: Park KH. Writing - review \& editing: Lim YM. Approval of final manuscript : all authors. 


\section{ORCID}

Yoon Min Lim https://orcid.org/0000-0002-9666-552X

Kwang Hyun Park https://orcid.org/0000-0002-5856-3928

Dong Won Lee https://orcid.org/0000-0003-0046-3139

Dae Hyun Lew https://orcid.org/0000-0002-2625-5664

Tai Suk Roh https://orcid.org/0000-0001-8681-159X

Seung Yong Song https://orcid.org/0000-0002-3145-7463

\section{REFERENCES}

1. Panettiere P, Marchetti L, Accorsi D, et al. Aesthetic breast reconstruction. Aesthetic Plast Surg 2002;26:429-35.

2. Baeke JL. Breast deformity caused by anatomical or teardrop implant rotation. Plast Reconstr Surg 2002;109:2555-64.

3. Brown MH, Shenker R, Silver SA. Cohesive silicone gel breast implants in aesthetic and reconstructive breast surgery. Plast Reconstr Surg 2005;116:768-79.

4. Heden P, Jernbeck J, Hober M. Breast augmentation with anatomical cohesive gel implants: the world's largest current experience. Clin Plast Surg 2001;28:531-52.

5. Hahn M, Kuner RP, Scheler P, et al. Sonographic criteria for the confirmation of implant rotation and the development of an implant-capsule-interaction ("interface") in anatomically formed textured breast implants with texturised Biocell-surface. Senologie-Zeitschrift für Mammadiagnostik und-therapie 2009;6:48-53.

6. Hammond DC, Migliori MM, Caplin DA, et al. Mentor Contour Profile Gel implants: clinical outcomes at 6 years. Plast Reconstr Surg 2012;129:1381-91.

7. Maxwell GP, Van Natta BW, Murphy DK, et al. Natrelle style 410 form-stable silicone breast implants: core study results at 6 years. Aesthet Surg J 2012;32:709-17.

8. Maxwell GP, Scheflan M, Spear S, et al. Benefits and limitations of macrotextured breast implants and consensus recommendations for optimizing their effectiveness. Aesthet Surg J 2014;34:876-81.

9. Maxwell GP, Van Natta BW, Bengtson BP, et al. Ten-year results from the Natrelle 410 anatomical form-stable silicone breast implant core study. Aesthet Surg J 2015;35:145-55.

10. Bengtson BP, Van Natta BW, Murphy DK, et al. Style 410 highly cohesive silicone breast implant core study results at 3 years. Plast Reconstr Surg 2007;120(7 Suppl 1):40S-48S.

11. Cunningham B. The Mentor study on Contour Profile Gel silicone MemoryGel breast implants. Plast Reconstr Surg 2017;120:33S-39S.

12. Montemurro P, Papas A, Heden P. Is Rotation a concern with anatomical breast implants? A statistical analysis of factors predisposing to rotation. Plast Reconstr Surg 2017;139:136778.

13. Heitmann C, Schreckenberger C, Olbrisch RR. A silicone implant filled with cohesive gel: advantages and disadvantages. Eur J Plast Surg 1998;21:329-32.

14. Brink RR. Sequestered fluid and breast implant malposition. Plast Reconstr Surg 1996;98:679-84.

15. Panettiere P, Marchetti L, Accorsi D. Rotation of anatomic prostheses: a possible cause of breast deformity. Aesthetic Plast Surg 2004;28:348-53.

16. Sampaio Goes JC. Breast implant stability in the subfascial plane and the new shaped silicone gel breast implants. Aesthetic Plast Surg 2010;34:23-8.

17. Pandya AN, Dickson MG. Capsule within a capsule: an unusual entity. Br J Plast Surg 2002;55:455-6.

18. Brody GS, Deapen D, Taylor CR, et al. Anaplastic large cell lymphoma occurring in women with breast implants: analysis of 173 cases. Plast Reconstr Surg 2015;135:695-705.

19. Department of Health Therapeutic Goods Administration (TGA) of Australian Government. Breast implants: update on TGA monitoring of anaplastic large cell lymphoma [Internet]. Symonston: TGA; c2017 [cited 2019 Jun 27]. Available from: https://www.tga.gov.au/alert/breast-implants-update-tgamonitoring-anaplastic-large-cell-lymphoma.

20. Barone FE, Perry L, Keller T, et al. The biomechanical and histopathologic effects of surface texturing with silicone and polyurethane in tissue implantation and expansion. Plast Reconstr Surg 1992;90:77-86.

21. Smahel J, Hurwitz PJ, Hurwitz N. Soft tissue response to textured silicone implants in an animal experiment. Plast Reconstr Surg 1993;92:474-9.

22. Derby BM, Codner MA. Textured silicone breast implant use in primary augmentation: core data update and review. Plast Reconstr Surg 2015;135:113-24.

23. Danino AM, Basmacioglu P, Saito S, et al. Comparison of the capsular response to the Biocell RTV and Mentor 1600 Siltex breast implant surface texturing: a scanning electron microscopic study. Plast Reconstr Surg 2001;108:2047-52. 